\title{
Robust System for Indoor Localisation and Identification for the Health Care Environment
}

\author{
Antti Ropponen · Henry Rimminen · Raimo Sepponen
}

Published online: 30 November 2010

(C) The Author(s) 2010. This article is published with open access at Springerlink.com

\begin{abstract}
In this study, an improved version of the low-frequency indoor location system, with a larger detection range and more durable antenna laminate, is presented. The basic system uses quad antennas, placed under the floor surface, to locate tags with $125-\mathrm{kHz}$ radio signals. The improvements were achieved with a one-layer laminate construction and transmitter electronics that can feed larger currents to the antennas. The measured tag detection height was $2 \mathrm{~m}$, which is adequate for location applications. The low-frequency signal was not affected by normal objects. The tag location reliability of $96.3 \%$ was verified with a practical test.
\end{abstract}

Keywords Indoor location · Low frequency $\cdot$ Near field imaging $\cdot$ Signal penetration

\section{Introduction}

Pervasive and context-aware computing often requires information about the locations and trajectories of people in an indoor environment where GPS, or related positioning, is not available [1]. Applications vary from interactive environments to health care informatics. To satisfy this need, different types of indoor location systems have been created in recent years. The location methods can be roughly divided into active and passive ones.

Passive methods observe the person's body itself. This can be done using cameras [2,3], measuring the static $[4,5]$ or dynamic [6] weight distribution, or measuring the body capacitance [7-10]. These methods produce very high tracking accuracies $(18-41 \mathrm{~cm}[3,10,13])$; however, no identification takes place. Remote biometric identification with passive methods

\footnotetext{
A. Ropponen $(\varangle) \cdot$ H. Rimminen $\cdot$ R. Sepponen

Department of Electronics, Aalto University, PO BOX 13340, 00076 Aalto, Finland

e-mail: antti.j.ropponen@tkk.fi

H. Rimminen

e-mail: henry.rimminen@tkk.fi

R. Sepponen

e-mail: raimo.sepponen@tkk.fi
} 
is possible $[4,5,11]$, but these systems must be trained before use. At close ranges facial recognition with computer vision is possible, but it requires near-full-frontal images of the person's face and cannot handle crowd shading [12].

In active methods the person carries a transponder that can be located using IR [13], visible light [14], ultrasound [15-18], or RF signals [19,20]. The advantage of the active methods is that identification is also provided. However, there are problems with the positioning accuracy. Excluding ultrasonic systems, active systems can seldom produce an error of less than $1.6 \mathrm{~m}[13,14,19,20]$. In some cases this is not enough to determine if a person is inside a certain room or on a certain floor. Proximity-based RFID positioning is also possible [21,22]. The accuracy of these methods lies within $0.5-1 \mathrm{~m}$, but they are strongly point-oriented: one RFID transponder is needed for each location [1]. Ultrasonic systems reach accuracies below $10 \mathrm{~cm}[15,18]$; however, acoustic signals are strongly attenuated by clothing and textiles [23]. Furthermore, the attenuation of clothing increases when the angle of incidence between the textile and the wave increases [24]. To attain reliable positioning, the ultrasonic transponder should be worn in such a way that it is visible.

In this study we present a method which combines the use of a passive system with high accuracy $[9,10]$ and an active system providing the identification [33]. The advantage of the proposed method is that the transponder is not disturbed by clothing, body shading, or being placed in a wallet or briefcase, unlike transponders using ultrasound, visible light, or IR. This is because the transmission uses magnetic fields. Furthermore, the passive and the active system can work both together and separately. In this study we use the positioning capability of an existing passive system called ELSI (Electronic Sensor with Intelligence), which uses NFI technology (Near Field Imaging) [9]. The system senses the conductivity of the human body by emitting a low-frequency electrical field. The field is produced and sensed by using a matrix of planar electrodes under the floor surface. The electrodes are etched on aluminium foil and are laminated between thin PET plastic films [9]. The location and trajectory of a person can be determined with a mean error of $21 \mathrm{~cm}$, and two people can be separated with $90 \%$ accuracy when the gap is $80 \mathrm{~cm}$ [25]. The NFI system is in actual use in a nursing home in Finland, where it detects residents falling, getting out of bed, exiting rooms, and entering the toilet [26].

The problem with the NFI system is that it cannot identify the person or object that has been located. This would be useful when falls occur or when patients suffering from dementia leave their rooms. Furthermore, nurses can also benefit from the identification feature as they could receive context-related information, such as patient records, or acknowledge NFI alarms by simply entering a room. Similar approaches have been used for example in automatic museum tour guides [27,28]. Identification could also be used for the positioning of ward equipment. Other applications could include interactive environments [29,30].

In this paper we implement an active RFID system (Radio Frequency Identification) to collaborate with the NFI system. The idea and the concept have been described in detail in our earlier work [33]. The RFID system uses quad antennas, which are placed on the same elements as the NFI sensor electrodes and active RFID transponders [31]. The RFID system maps the tags using low-frequency fields $(125 \mathrm{kHz})$ transmitted with the quad antennas. The tag then uses high-frequency radio $(2.4 \mathrm{GHz})$ to send its identification number and received location data that are received back to the system. The tag used tag is active because the read range of passive tags is not adequate at LF frequency [32]. The read range of passive UHF tags is adequate, but UHF transmitter antennas could not be embedded in the floor because of long wires and unpredictable dielectric properties [33].

Our previous attempts at integrating RFID quad antennas with NFI elements showed that two-layer structures are expensive and vulnerable to mechanical wear. We also encountered 
problems with antenna power delivery, which resulted in an inadequate read range. We now illustrate how the quad antenna can be integrated with the NFI element using a single-layer structure. In addition, we present a high-current multiplexing technique, which makes possible the near-simultaneous operation of the RFID and NFI systems using the same sensor film. In this study we illustrate the performance of the system by performing a series of tests.

\section{Requirements}

The previous laminate had a complex structure. Both the NFI and RFID signal wires crossed each other, and thus the laminate had two layers [33]. The two-layered structure had major disadvantages. The joints between the two metallic layers were very fragile and the manufacturing costs of the laminate were four times larger compared to those of single-layered ones. The structure also made it necessary to manufacture laminates in 7-m pieces. When the elements were placed in narrower rooms, the rest of the 7-metre-long laminate went to waste.

The problem with the previous multiplexer electronics (MUX) was that the RFID signal was routed to the antenna without amplification. The ADG706 multiplexer that was used could only hold out a 30- $\mathrm{mA}_{\mathrm{rms}}$ current with a square wave [34]. This yields $4.8 \mathrm{~mA}_{\mathrm{rms}}$ in the desired carrier frequency (sinusoidal waveform). At this current level, a mean receive height of $1.5 \mathrm{~m}$ was achieved with the RFID tag. This was not adequate for practical use. The inducted magnetic field of a quad antenna is directly proportional to its current. Hence a new version of the MUX electronics with a higher current output was needed.

To summarise, the main goals of the study were:

1) Design a laminate layout that has only one layer and can be manufactured as continuous pieces;

2) Design MUX electronics that make possible a receive height of $2 \mathrm{~m}$ using the existing tag design;

3) Determine the penetration capabilities of the low-frequency signal with different materials and objects.

\section{Materials and Methods}

\subsection{Laminate}

Possibly the most critical issue in the design of the laminate is the manner of wiring. The wiring takes up space from the NFI elements and reduces the area covered. On the other hand, there have to be many wires to attain long sensor laminates. Long laminates are required to cover large rooms.

To reduce the number of signal wires, it was decided that the NFI and the RFID systems would use the same signal wires on the laminate. In this way, only two wires would be needed instead of three to feed both the sensor plate and the quad antenna. The technique is illustrated in Fig. 1. Switch 1 selects the origin of the signal. If the signal comes from the NFI signal generator, Switch 2 is turned to the high-impedance state and the signal is fed to the NFI sensor. When the RFID signal is turned on, Switch 1 is connected accordingly and Switch 2 connects the return path. In this way the RFID signal is conducted to the quad antenna. 
Fig. 1 The same wires are used both for the NFI sensor and the RFID quad antenna

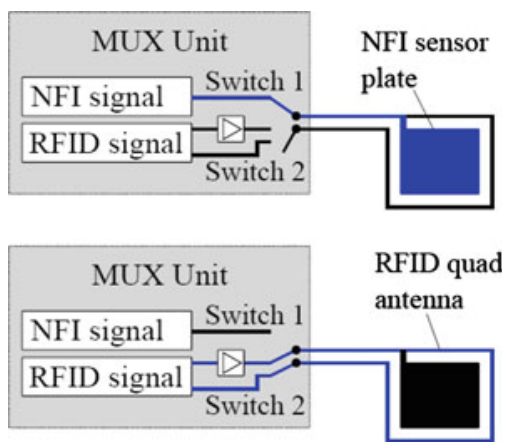

The signal wire is $8 \mu \mathrm{m}$ thick [9] and its skin depth $\delta$ can be calculated with Eq. 1 [35]:

$$
\delta=\sqrt{\frac{2}{\omega \mu \sigma}}
$$

where $\omega$ is the radian frequency $(2 \pi \times 125 \mathrm{kHz}), \mu$ is the conductor magnetic permeability $\left(\mu=\mu_{0}=1.26 \times 10^{-6} \mathrm{Vs} /(\mathrm{Am})\right.$ ), and $\sigma$ the bulk conductivity (aluminium, $37.8 \times 10^{6} \mathrm{~S} / \mathrm{m}$ ). With the given values the skin depth is $230 \mu \mathrm{m}$, and thus it is greater than the thickness of the wire. Hence the current density is homogenous over the entire cross-section of the conductor and the resistance of the wirings can be calculated with Eq. 2 [36]:

$$
R=\frac{\rho L}{t W}
$$

where $W$ is the width of the wire, $t$ is its thickness, $L$ is its length, and $\rho$ is its resistivity. Narrowing the signal wires reduces the area taken away from the NFI elements. However, as we can see from Eq. (2), the resistance then increases. And, with a constant voltage source, the current that flows through the antenna is inversely proportional to the impedance of the wirings and the antenna. The magnetic field that the antenna creates is directly proportional to the current in the antenna. Hence the current in the antenna was calculated using solely the dc resistance of the wires.

\subsection{Electronics}

The main purpose was to construct a new MUX unit which would have the switching capability shown in Fig. 1. The electronics should also be capable of amplifying the current after the multiplexers to gain larger magnetic fields. It should also be able to route the NFI and RFID signals to the right antennas as the previous units did.

\subsection{The Tag}

The tag used in this study was very similar to the one described in our earlier work [33], with the exception that an AS3931 was used as an LF receiver and a CC2430 was used as the ZigBee radio. The tag includes a 3-dimensional antenna, which was tuned to $125 \mathrm{kHz}$, an AS3931 receiver, and a CC2430 system-on-chip which includes a microcontroller and ZigBee radio. The tag is currently powered by a $3 \mathrm{~V}$ button cell. The dimensions of the tag were $80 \mathrm{~mm} \times 50 \mathrm{~mm} \times 10 \mathrm{~mm}$. 
The AS3931 receiver requires a preamble data burst to be awoken and receive data. An 8-bit Manchester coded preamble sequence with a bit rate of $1.4 \mathrm{kHz}$ and a carrier frequency of $125 \mathrm{kHz}$ was used. A modified FM0 encoding [32] was used for data transmission.

\section{Experiment Design}

\subsection{Detection Volume Test}

Our primary goal was to evaluate the coverage volume of one antenna. This included the measurement of the detection height directly above the antenna and its spatial distribution around it. An antenna in the centre of the room was selected so that there would be adequate clearance from the walls. The signal was fed only to that antenna and the tag described above was used for receiving. The ZigBee radio in the tag was used for transmitting the LF read values back to the system. The system consisted of a ZigBee coordinator and a PC that visualised and logged the data. In this way successful LF reception could be verified. Detection heights were measured at five different directions from the centre of the quad antenna $(+\mathrm{X},+\mathrm{Y},-\mathrm{X}$, diagonal $+\mathrm{X}+\mathrm{Y}$, and diagonal $-\mathrm{X}+\mathrm{Y})$; see Fig. 2. The tag was placed on a wooden rack that did not include any metallic parts. The rack was moved in $25-\mathrm{cm}$ steps in each direction. At each measurement point the highest read height where the tag could receive the preamble correctly 5 times in a row was recorded.

\subsection{Penetration Test}

The secondary goal test was how well the signal penetrates different objects. The tag was placed inside normal objects, for example a briefcase, to illustrate the robustness of data transmission based on a magnetic field. In the penetration test, the detection volume test was repeated with two exceptions: only one location directly above the antenna was used and different objects were placed between the transmitting antenna and the receiver. First the tag was placed inside a folded cotton shirt, a wallet, a plastic briefcase, and an antistatic bag (3M, SCC DRI-SHIELD ${ }^{\mathrm{TM}}$ 2000). One end of the antistatic bag was left open and facing upwards, allowing the ZigBee to work. The tag was also placed on top of a sheet of aluminium foil $(29 \mathrm{~cm} \times 38 \mathrm{~cm})$ and on a steel plate $(52 \mathrm{~cm} \times 33 \mathrm{~cm} \times 0.1 \mathrm{~cm})$ to demonstrate extreme conditions.

Fig. 2 The coverage volume measurement. The origin is in the middle of the transmitting antenna. The tag was placed on a wooden rack. The heights were measured on five different tracks

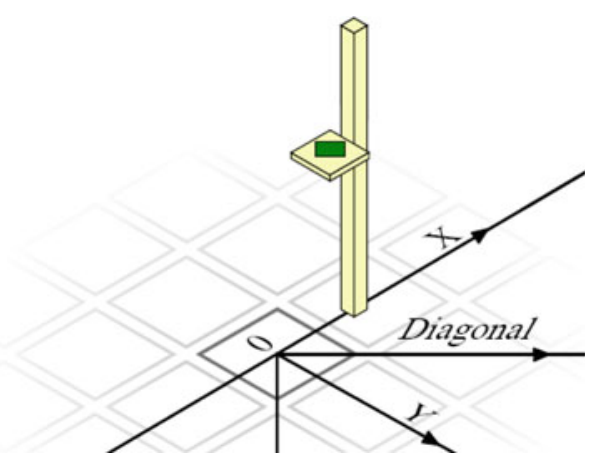




\subsection{Practical Test}

The operation of the system in realistic conditions was verified in the practical test. The test resembled the penetration test, but had multiple locations and a real person carrying the tag. In the tests, the same tag was used as the receiver. The tag was placed inside four different pieces of clothing or objects worn or carried by the person: the breast pocket of a shirt, the breast pocket of a jacket, a leather wallet in the jacket pocket, and a plastic briefcase that was carried in one hand. Also a crowded situation was tested by surrounding the person with four people in all locations. The tag was held in the test subject's breast pocket. The preamble sequence was transmitted to all the quad antennas in the test room in every test case. The criterion for a successful trial was a correctly received preamble sequence from at least one antenna. The trials were conducted in 20 randomly selected locations inside the test room. The locations were the same when the clothing/enclosure was changed.

\section{System Structure}

\subsection{Laminate}

The structure of the NFI laminate can be seen in Fig. 3. First of all, the structure has a continuous pattern. The green dashed lines show the spots where the element can be cut and in every spot the layout of the connectors remains the same. The wires are wider in the connector area in order to have a raster of $2.54 \mathrm{~mm}$. This enables a large variety of connectors to be used. Furthermore, with a large raster the attachment of the connector is easier. There are 32 connectors altogether. Thirty of them are signal wires to the antennas and sensors. The two outermost are ground wires used for the NFI measurement. All the corners are rounded to avoid fragile sharp edges.

To reduce the number of signal wires, only every other NFI element was surrounded with an antenna. Because of the nearly spherical radiation pattern of a small quad, it is not necessary to have an antenna on every NFI element [33]. With the targeted 2-m receive height the read distance is almost constant in a horizontal plane. Hence different fields overlap with each other, even with this raster. Antennas that are connected together with NFI plates and their wires are denoted with the colour blue in Fig. 3. In these plates the RFID antennas

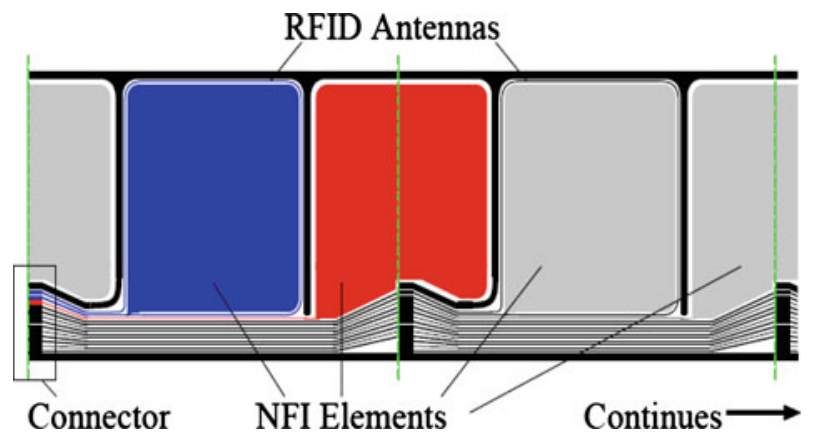

Fig. 3 The NFI-RFID element. Blue wires go to both the NFI element and the RFID antenna. The red wire goes to a single NFI plate. The laminate has a continuous structure. The dashed lines show the places where the laminate can be cut. The wirings and laminate layout remain the same, irrespective of which dashed line is selected 
and NFI sensors use common signal wires. The NFI plates and wires without an antenna are denoted with the colour red. With this structure, only three wires are needed for one quad antenna and two sensor plates. The signal wires that lead to the quad antennas are $1.8 \mathrm{~mm}$ wide because of the high currents. The NFI sensor wires are $0.6 \mathrm{~mm}$ wide because of the significantly lower current demand.

In our previous version of the NFI element the signal was fed to one NFI sensor plate and the neighbouring plates were grounded. Hence the signal coupled from the transmitting sensor to the grounded plates [9]. However, when the NFI and RFID signals had common signal wires the grounding was not possible. That is why separate ground wires were added to the laminate so that the NFI can use them as a reference potential (ground). The thick black wires in Fig. 3, which surround the sensor plates and the lowermost wire, act as the reference potential.

\subsection{Test Room}

A new test room was built to pilot the new laminates and the electronics. The room had a $9 \times 15$ NFI sensor matrix with a $9 \times 8$ antenna matrix embedded in it. The dimensions of the room were $6.4 \mathrm{~m} \times 4.6 \mathrm{~m}$. The test room is located in the Department of Electronics, Aalto University, Espoo, Finland. The existing floor of the room had severely worn linoleum flooring, with large areas having bare concrete visible. The existing floor was first levelled out with 22-mm chipboard panels, to which the sensor laminates were attached. The laminates were then covered with 3- mm PVC flooring. The adhesive used was Cascoflex, by Akzo Nobel Oy, Finland. In Fig. 4 the floor is shown just before the surface was installed.

\subsection{Electronics}

Figure 5 illustrates the system that was built. The main unit selects the MUX unit and the NFI plate or the RFID antenna, where the signal is fed. It also amplifies the RFID signals. The multiplexer units route the signals to the NFI elements and to the RFID antennas. Up

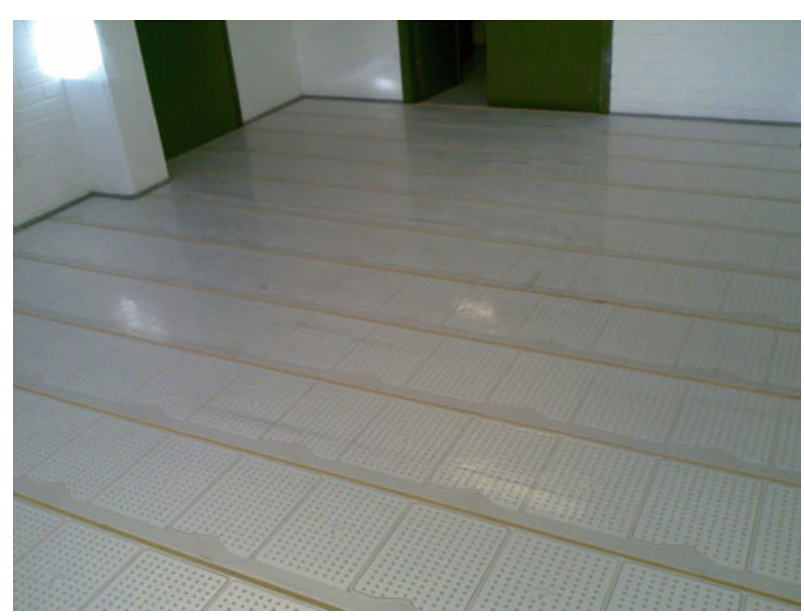

Fig. 4 The floor of the test room was covered with the laminate that had been developed 


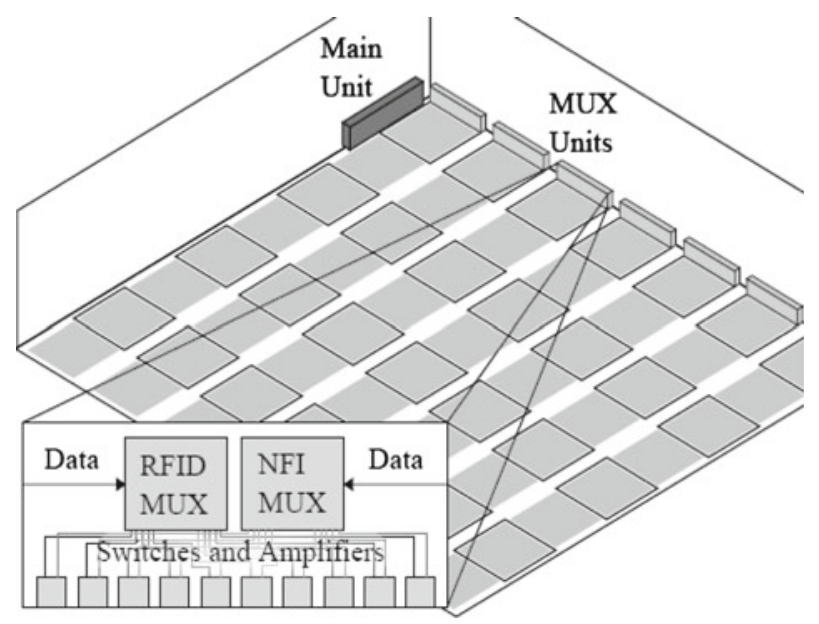

Fig. 5 System structure. Data, which consist of routing addresses and the transmitted signal, come from the main unit. The MUX units consist of multiplexers, switches, and RFID signal amplifiers

Fig. 6 Switching and current amplifier circuit. When the NFI signal is transmitted the RFID input is in high-impedance state, and vice versa

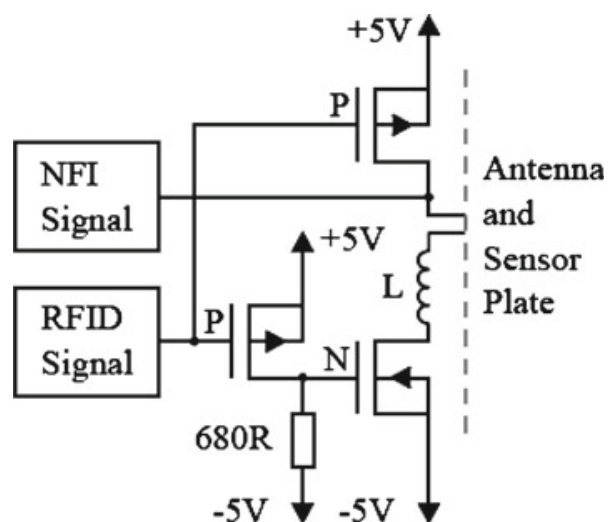

to 16 MUX units can be linked together. One MUX unit includes one ADG1206 and one ADG1208 low-capacitance multiplexer. The RFID signals are fed using a 74HC154 digital decoder and 10 switching/amplifying units; see Fig. 5. One MUX unit can then route the signals to 20 NFI plates and 10 RFID antennas (every other plate is surrounded with the antenna).

The switching between the NFI and RFID signals is performed with MOS transistors (Fig. 6). The benefits of the MOS transistors are that they can hold out rather large currents, their drain-source resistance is small, and the whole switch fits into a very small area (10 $\mathrm{mm} \times 10 \mathrm{~mm}$ ). When the RFID signal source is set to $+5 \mathrm{~V}$, the PMOS transistors are in an OFF state. This also sets the NMOS transistor to the OFF state, because its gate voltage goes to $-5 \mathrm{~V}$. In this state the NFI signal sees high impedance to the antenna and low impedance to the sensor plate. When the RFID signal is transmitted, the NFI signal source is set to a high-impedance state so that that the RFID signal does not harm the NFI multiplexer. The RFID signal is created simply by switching the PMOS transistors to their ON and OFF states, creating ASK modulation. 


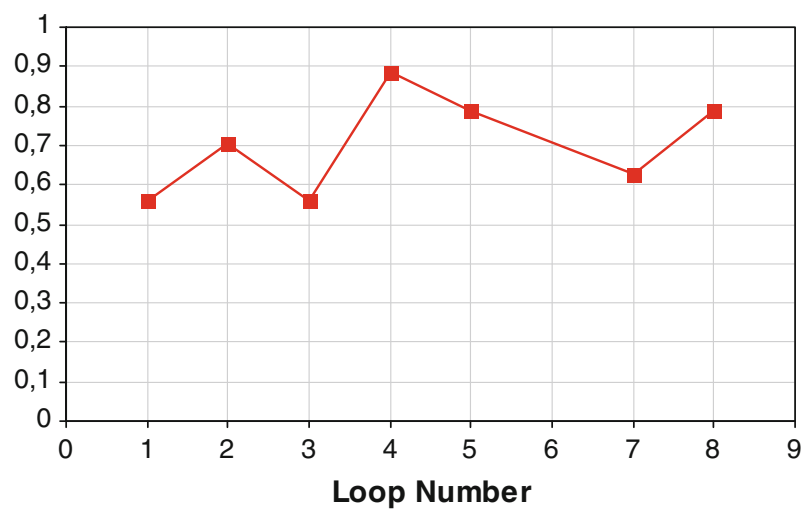

Fig. 7 Normalised magnetic field of one row of compensated quad antennas (linear scale)

Because the signal wires of the different antennae had different resistances, the current had to be compensated to create magnetic fields that were as homogenous as possible. Without that, the magnetic field in a loop next to the MUX unit would have been significantly higher than the one in the tenth loop. The problem with the power resistors was that they are too big to fit in a $1-\mathrm{cm}^{2}$ area. The problem was solved using a power inductor. 0.805 cased inductors could hold out the targeted $100 \mathrm{~mA}_{\mathrm{rms}}$ current with a square wave.

The DC resistances of one row of antennas were measured in the test room. They increased linearly from $3 \Omega$ in the first loop to $30 \Omega$ in the eighth loop. The inductance of an antenna at the centre of the room was $3.7 \mu \mathrm{H}$. This inductance causes a reactance of $2.9 \Omega$ with the $125-\mathrm{kHz}$ transmitting frequency and was considered negligible compared to the resistances found. The compensation inductors were chosen to be such that the absolute values of the antenna impedances remained approximately the same. The targeted impedance was $30 \Omega$, which was equal to the greatest antenna resistance. With a $10-\mathrm{V}$ power supply we achieved the targeted $100 \mathrm{~mA}_{\mathrm{rms}}$ current.

The magnetic fields generated by the quad antennas were measured with a Rohde \& Schwarz ESCS30 EMI test receiver. The normalised magnetic field strengths of one row of antennas can be seen in Fig. 7. They were measured at the centres of the antennas at the floor surface.

\subsection{Experiment Result}

\subsubsection{Detection Volume Test}

The results of the detection volume test can be seen in Fig. 8. On the left (a) the detection height is presented in relation to the distance from the origin on three axes. On the right (b) all the measured values are illustrated with a 3D plot.

\subsubsection{Penetration Test}

The results of the penetration test can be seen in Table 1 . The read heights were measured in the same way as in the detection volume test, but only directly above the antenna. First the height was measured without any obstructions, and then it was repeated with six different obstacles. 

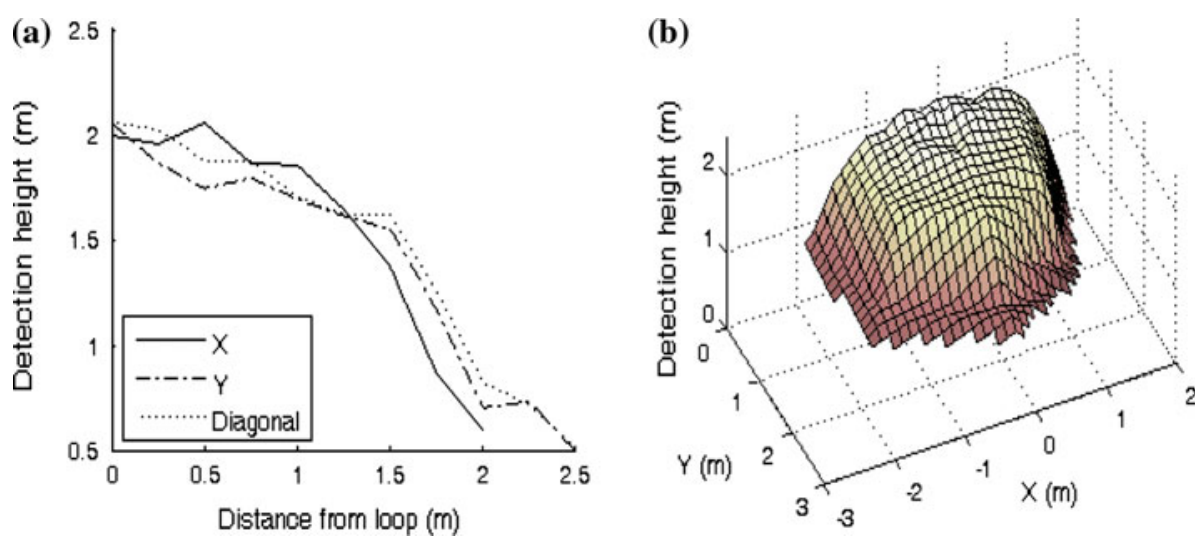

Fig. 8 Detection volume of a single quad antenna at the centre of the room. a Results of three measurement axes. The labels in the legend refer to Fig 2. b 3D surface plot containing all measurements. The antenna is in the origin

Table 1 Penetration of the RFID signal

\begin{tabular}{ll}
\hline & Read height $(\mathrm{cm})$ \\
\hline No obstructions & 200 \\
Folded in a shirt & 200 \\
In a wallet & 200 \\
In a briefcase & 200 \\
In an antistatic bag & 200 \\
On an aluminium foil sheet & 110 \\
On a steel plate & 85 \\
\hline
\end{tabular}

Read height is measured directly above the antenna

Table 2 The practical test

\begin{tabular}{llllll}
\hline Location & Shirt pocket & Jacket pocket & Wallet & Briefcase & Crowded \\
\hline Success & $19 / 20$ & $19 / 20$ & $19 / 20$ & $20 / 20$ & $19 / 20$ \\
\hline
\end{tabular}

\subsubsection{Practical Test}

The results of the practical test can be seen in Table 2. The proportions represent the rate of correct transmissions in 20 different locations in the room. The locations were randomly selected. We found that the ZigBee radio gave perfect coverage of the test room.

\section{Discussion}

As a result of this study we implemented a circuitry and conductor geometry which enables the existing NFI location system [9] and the add-on RFID system [33] to be operated in a near-simultaneous manner.

The proposed implementation produced relatively even magnetic fields when the antennas in one row were compared (cross-section of the room). This reduces the need for 
(a)

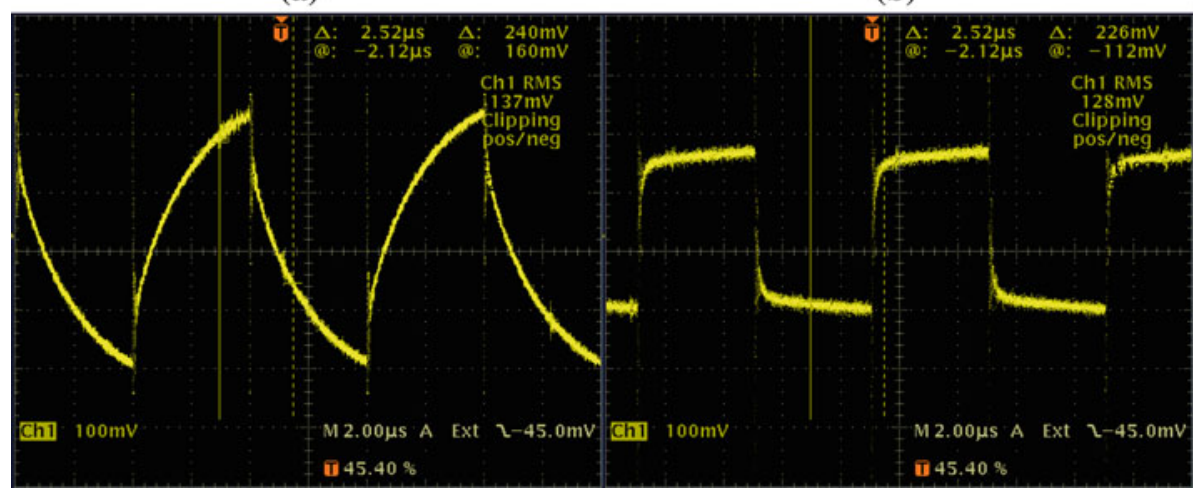

Fig. 9 Oscilloscope images of currents in equivalent circuits of a The first antenna; b The eighth antenna

compensation coefficients for each antenna. Calculated from Fig. 7, the field strengths have an $18 \%$ standard deviation. The deviation was caused by two factors: the compensation inductors were chosen from a discrete set of inductance values and the use of inductors modified the waveforms in the antennas. Figure 9 shows the estimated waveforms flowing though the first and the eighth antenna. They were measured by using an equivalent circuit, which took into account the resistivity and the parasitic capacitance of the wires. A $1-\Omega$ series resistor was used for current-voltage transformation, and the signal was fed by a signal generator with a $10 \mathrm{~V}_{\mathrm{pp}}$ square wave output. The rms currents are approximately the same (1st: $137 \mathrm{~mA}$, 8th: $128 \mathrm{~mA}$ ), but the waveform in the first antenna resembles a triangle wave and the one in the eighth antenna resembles a square wave. This, together with the quantised inductance values, explains the difference in the magnetic fields.

As can be seen from Fig. 8, a 2-m receive altitude was achieved in the detection volume test, and hence the targeted value was reached. The receive height of $2 \mathrm{~m}$ is considered to be more than adequate for person-tracking purposes. The shape of the detection volume of a single antenna is nearly spherical. The reason for the slight variation is that the antenna is not a square but a rectangle (Fig. 3). Having rotationally symmetrical radiation patterns and having relatively even spatial field strengths creates a solid base for locating the tag.

We suspect that the location can be calculated by using the centroid of all the received antenna locations.

The penetration tests showed that normal objects hardly affect the system at all; see the first five rows in Table 1. The steel plate and the aluminium foil affected the receive height strongly, as expected. When the wave confronts the material orthogonally it attenuates as:

$$
-20 \log \left(\frac{1}{e}\right)^{\frac{t}{\delta}},
$$

where $t$ is the thickness and $\delta$ the skin depth of the material. The skin depth can, as previously mentioned, be calculated with Eq. 1. Calculated values can be seen in Table 3. From the values (Table 3 ) we can see how the steel plate attenuates the signal drastically. This in itself could explain how the read height is so low in the penetration test (Table 1). However, the attenuation does not explain why the read height results with the thin aluminium foil were also low (Table 1). Hence the penetration tests were repeated, first with the tag placed on top of the plates and then with it under the plates. With this arrangement the way in which the 
Table 3 Calculated penetration attenuations of different objects

\begin{tabular}{lllll}
\hline & $\sigma(\mathrm{S} / \mathrm{m})$ & $t(\mu \mathrm{m})$ & $\delta(\mu \mathrm{m})$ & Attenuation $(\mathrm{dB})$ \\
\hline Steel plate & $10.4 \times 10^{6}$ & 1.000 & 441 & 19 \\
Aluminium foil & $37.6 \times 10^{6}$ & 11 & 230 & 0.4 \\
Antistatic bag & $37.6 \times 10^{6}$ & $2 \times 0.003$ & $2 \times 230$ & $\sim 0$ \\
\hline
\end{tabular}

Table 4 Repeated penetration test

The tag was first placed on top of the obstructive object, then under it

\begin{tabular}{lcl}
\hline & Read height/on $(\mathrm{cm})$ & Read height/under $(\mathrm{cm})$ \\
\hline Steel plate & 84 & 107 \\
Aluminum foil & 110 & 113 \\
\hline
\end{tabular}

circulating currents affect the receiving antenna was shown. As we can see from Table 4, the read height increases with the steel plate when the tag is placed under it. However, there is almost no increase in the read height with the aluminium foil. If the attenuation only affected the read height, the plates above the tag would not have any impact on the results.

Shading and circulating currents caused by metallic objects may be relevant if the tag is used to track, for example, a steel equipment trolley. The tag has to be then placed either near the floor or far enough from the steel plates.

As we can see from the result of the practical test (Table 2), the tag can receive the preamble in $97 \%$ of the cases. The reason why the result was not $100 \%$ was that around two locations there was a connector failure in the laminate, which could not be repaired. In the blind spots the NFI system could locate the person but identification was lacking. However, from the results we can see that the system is robust for detecting if a tag is in the service area, even if the tag is inside common objects. A human body does not either affect the signal nearly at all. These may be a problem if a location system based on ultrasound, IR, or visible light is used.

The laminate fulfilled the requirements well. A good compromise between the number and width of the wirings for this system were achieved. The only problem was that some of the antennas broke down. The reason was in the connectors crimped on to the laminates. Apparently aluminium as a conducting material is not suitable for a crimp connection, since it creates an insulating oxide when in contact with air.

\section{Conclusions and Future Work}

On the basis of the results we conclude that the system provides a robust way to transmit information from conductor structures in the floor to a tag carried by a person. The detection range is good and it is not affected by clothing, body shading, or placement in a dielectric enclosure.

Our future work includes the measurement of the positioning accuracy and tracking capabilities of the RFID system together with the NFI system. This includes multi-target separation. In future installations we will enclose the base of the laminate connector in epoxy to prevent oxide from forming and consider changing the conductor material to copper. The problem with the triangular waveform could be solved and more even field strengths could 
be achieved if a constant current source were used instead of the compensation inductors. This is also considered in our future designs.

Open Access This article is distributed under the terms of the Creative Commons Attribution Noncommercial License which permits any noncommercial use, distribution, and reproduction in any medium, provided the original author(s) and source are credited.

\section{References}

1. Hightower, J., \& Borriello, G. (2001). Location systems for ubiquitous computing. Computer, 34(8), 57-66.

2. Krumm, J., Harris S., Meyers, B., Brumitt, B., Hale, M., \& Shafer, S. (2000). Multi-camera multiperson tracking for easyLiving. In Proceedings of the third IEEE international workshop on visual surveillance (VS'2000).

3. Harville, M. (2004). Stereo person tracking with adaptive plan-view templates of height and occupancy statistics. Image Vision Computer, 22(2), 127-142.

4. Addlesee, M. D., Jones, A., Livesey, F., \& Samaria, F. (1997). The ORL active floor. IEEE Personal Communications, 5(4), 35-41.

5. Orr, R. J., \& Abowd, G. D. (2000). The smart floor: A mechanism for natural user identification and tracking. In Conference on human factors in computing systems (CHI 2000) (pp. 1-6). The Hague: Netherlands (April).

6. Lekkala, J., \& Paajanen, M. (1999). EMFi-new electret material for sensors and actuators. In Proceedings of the IEEE. 10th international symposium on electrets (pp. 743-746).

7. Zimmerman, T. G. (1996). Personal area networks: Near-field intrabody communication. IBM Systems Journal, 35(3 \& 4), 609-617.

8. Savio, D. \& Ludwig, T. (2007). Smart carpet: A footstep tracking interface. In Proceedings 21st International conference on advanced information networking and applications workshops, AINAW '07 (pp. 754-760). Niagara Falls: Canada.

9. Rimminen, H., Linnavuo, M., \& Sepponen, R. (2008). Human tracking using near field imaging. In 2nd International conference on pervasive computing technologies for healthcare.

10. Valtonen, M., Mäentausta, J., \& Vanhala, J. (2009). Tiletrack: Capacitive human tracking using floor tiles. In 7th Annual IEEE international conference on pervasive computing and communications (PerCom 2009).

11. BenAbdelkader, C., Cutler, R., \& Davis, L. (2002). Stride and cadence as a biometric in automatic person identification and verification. In Proceedings fifth IEEE international conference on automatic face and gesture recognition (pp. 372-377). Washington, D.C. (May 21 2002).

12. Williams, M. (2007). Better face-recognition software. Technology Review (30 May 2007).

13. Want, R., Hopper, A., Falcao, V., \& Gibbons, J. (1992). The active badge location system. ACM Transaction on Information Systems, 10(1), 91-102.

14. Cheok, A. D., \& Li, Y. (2008). Ubiquitous interaction with positioning and navigation using a novel light sensor-based information transmission system. Personal and Ubiquitous Computing, 12(6), 445-458.

15. Priyantha, N. B. (2005). The cricket indoor location system, Doctoral Thesis. Massachusetts institute of technology: Department of Electrical Engineering and Computer Science (June 2005).

16. Hazas, M., \& Ward, A. (2002). A novel broadband ultrasonic location system, Lecture notes in computer science. Springer Berlin:Heidelberg (2498/2002, Jan 2002).

17. Hori, T., Nishida, Y., Aizawa, H., Murakami, S., \& Mizoguchi, H. (2004). Sensor network for supporting elderly care home. Proceedings of IEEE Sensors, 2, 575-578.

18. Harter, A., Hopper, A., Steggles, P., Ward, A., \& Webster, P. (2002). The anatomy of a context-aware application. Wireless Networks, 2(2), 187-197.

19. Hii, P., \& Zaslavsky A. (2005). Improving location accuracy by combining WLAN positioning and sensor technology, Workshop on real-world wireless sensor networks (REALWSN'O) pp. 20-21. Stockholm: Sweden (June).

20. Cho, H., Kang, M., Park, J., Park, B. \& Kim, H. (2007). Performance analysis of location estimation algorithm in zigBee networks using received signal strength. In Proceedings 21st international conference on advanced information networking and applications workshops (AINAW'07) (pp. 302-306). Niagara Falls: Canada (May 21-23). 
21. Hihnel, D., Burgard, W., Fox, D., Fishkin, K., \& Philipose, M. (2004). Mapping and localization with LFID technology. In Proceedings IEEE international conference on robotics \& Automation (pp. 1015-1020) New Orleans, LA (April).

22. Willis, S. \& Helal, S. (2005). LFID information grid for blind navigation and wayfinding. In Proceedings ninth IEEE international symposium on wearable computers (ISWC'05) (pp. 34-37). Osaka, Japan (Oct. 18-21).

23. Blomme, E., Bulcaen, D., Declercq, F., \& Lust, P. (2002). Air-coupled ultrasonic evaluation of coated textiles. In Ultrasonics symposium, 2002. Proceedings. 2002 IEEE , 1, 757-760 (8-11 Oct. 2002).

24. DuChateau, J. E. (2005). Using ultrasound in concealed weapons detection, senior Thesis. NDE Lab, Department of Applied Science, College of William and Mary: Williamsburg, VA (pp. 15-20). (April 2005).

25. Rimminen, H., Lindström, J., \& Sepponen, R. (2009). Positioning accuracy and multi-target separation with a human tracking system using near field imaging. International Jornal on Smart Sensing and Intelligent Systems, 2(1), 156-175.

26. Oksanen, R., Paldanius, S., Nykänen, J., Linnavuo, M., Raivio, K., Segerstam, K. E., Sepponen, R., Pohjola, L., \& Finne-Soveri, H. (2009). Testing and adopting floor-sensor solutions in daily practice for patient safety in Kustaankartano nursing home. The Journal of Nutrition, Health and Aging, 13 (supplement 1), 361 (Jul. 2009 [abstract]) and at 19th IAGG world congress of gerontology and geriatrics Paris, France 2009 [poster].

27. Abowd, G. D., Atkeson, C. G., Hong, J., Long, S., Kooper, R., \& Pinkerton, M. (1997). Cyberguide: A mobile context-aware tour guide. Wireless Networks, 3(5), 421-433.

28. Thrun, S., Bennewitz, M., Burgard, W., Cremers, A. B., Dellaert, F., Fox, D., Hähnel, D., Rosenberg, C., Roy, N., Schulte, J., \& Schulz, D. (1999). MINERVA: A second-generation Museum tour-guide robot. Proceedings-IEEE International Conference on Robotics and Automation, 3, 1999-2005.

29. Linnavuo, M., \& Rimminen, H. (2010). Localization and monitoring of people with a near-field imaging system: Boosting the elderly care. In Pervasive and smart technologies for healthcare: Ubiquitous methodologies and tools pp. 78-96.

30. Linnavuo, M., Ojapelto, A., \& Sepponen, R. (2009). A proactive space for rehabilitation, gaming and multimodal interaction: A poster presentation. In Proceedings of the 13th international mindTrek conference: Everyday life in the Ubiquitous Era (p. 213).

31. Finkenzeller, K. (2003). RFID Handbook-fundamentals and applications in contactless smart cards and identification Second Edition. John Wiley \& Sons.

32. Dobkin, D.M. (2008). The RF in RFID: Passive UHF RFID in practice (p. 25). Burlington, MA: Elsevier Inc. (p. 72).

33. Ropponen, A., Linnavuo, M., \& Sepponen, R. (2009). LF indoor location and identification system. International Journal on Smart Sensing and Intelligent System, 2(1), 94-117 (March 2009).

34. Analog Devices ADG706/ADG707 datasheet. (2002). Analog Devices Inc: Norwood, Maine (2002).

35. Rautio, J. C., \& Demir, V. (2003). Microstrip conductor loss models for electromagnetic analysis. IEEE Transactions on Microwave Theory and Techniques, 51(3), 915-921.

36. Johns, D. A., \& Martin, K. (1997). Analog integrated circuit design. John Wiley and Sons, Inc.

\section{Author Biographies}

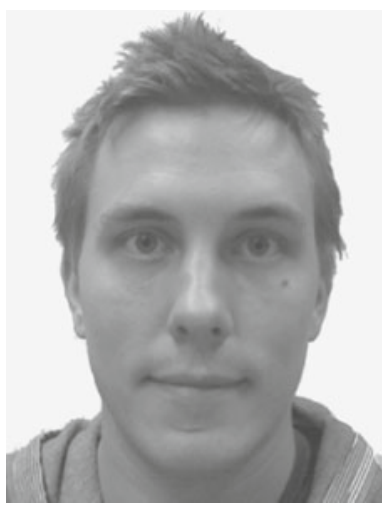

Antti Ropponen received his M.Sc. (Tech.) degree in 2007 in telecommunications engineering from the Helsinki University of Technology, Espoo Finland. He has worked as development engineer at Vesatel private limited company. His present position is Research Scientist at the Applied Electronics Research unit, Aalto University, School of Science and Technology, Espoo, Finland. His main interests are RFIDtechnology, low-power networks and mobile devices. He is a member of the Finnish Society of Electronics Engineers. 

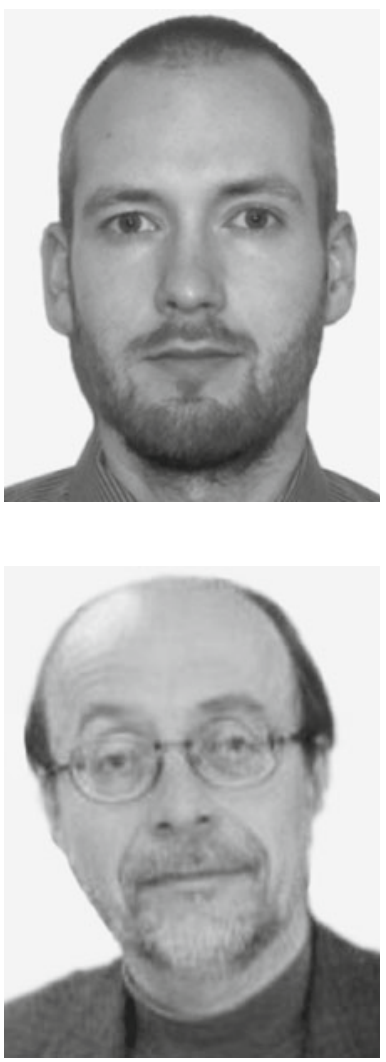

Henry Rimminen received his M.Sc. (Tech.) degree in electrical engineering in 2006 from Helsinki University of Technology, Espoo, Finland. He is currently working as a Researcher at the Applied Electronics Laboratory, Department of Electronics, Aalto University, Espoo Finland. His main interests are embedded systems and capacitive sensors. He is a member of the Finnish Society of Electronics Engineers and the Finnish Association of Graduate Engineers.

Raimo Sepponen received his D.Sc. degree in electrical engineering from the Helsinki University of Technology, Espoo, Finland, in 1986. He has worked in Instrumentarium Corporation where he developed MRI systems. In 1994 he joined the Helsinki University of Technology, as a professor of Applied electronics. His present position is Head of department in Aalto University, School of Science and Technology, Department of Electronics. He has authored more than 50 scientific papers and received patents worldwide related to more than 25 inventions. Dr. Sepponen has received the Award of Engineering from the Finnish Engineering Society, the Award of most significant doctoral thesis in Radiology from the Nordic Radiological Society, and the Award for development of MRI from the European Society of Magnetic Resonance Imaging. 